\title{
Çok Antenli Bilişsel Radyolarda GLRD Tabanlı Spektrum Algılama**
}

\author{
Meryem Yarar ${ }^{1}$, Fatih Yavuz Ilgın², Cebrail Çiflikli ${ }^{3}$ \\ ${ }^{1}$ Erzincan Binali YILDIRIM Üni, Fen Bilimleri Ens.Elektrik Elektronik Mühendisliği ABD Yüksek Lisnas Öğrencisi, Erzincan, Türkiye \\ ${ }^{2}$ Erzincan Binali YILDIRIM Üniversitesi, Mühendislik Fakültesi, Elektrik Elektronik Müh. Bölümü, Erzincan, Türkiye (ORCID: 0000-0002-7449-4811) \\ ${ }^{3}$ Kayseri Üniversitesi, Meslek Yüksekokulu, Elektrik ve Otomasyon Bölümü, Kayseri, Türkiye (ORCID: 0000-0002-7449-4811)
}

(Konferans Tarihi: 5-7 Mart 2020)

(DOI: 10.31590/ejosat.araconf36)

ATIF/REFERENCE: Yarar, M., Ilgın, F. Y \& Çiflikli, C. (2020). Çok Antenli Bilişsel Radyolarda GLRD Tabanlı Spektrum Algılama. Avrupa Bilim ve Teknoloji Dergisi, (Özel Sayı), 285-291.

$\ddot{O} \mathbf{z}$

Bilindiği gibi, çok antenli Bilişsel Radyo sistemleri için özdeğer tabanlı spektrum algılama yöntemleri algılanacak sinyale ilişkin önceden hiçbir bilgi gerektirmemesi nedeni ile oldukça tercih edilen bir yöntemdir. Bunun yanısıra özdeğer tabanlı algılama yöntemleri genellikle gürültü belirsizliği faktöründen en az etkilenen yöntemlerdir. Özdeğer tabanlı algılama yöntemlerinde algılama performansı, test istatistiğinin doğru hesaplanmasına ve eşik değerine bağlıdır. Bu çalışmada genelleştirilmiş en çok olabilirlik tabanlı algılama(Generalized Likelihood Ratio Detection- GLRD) yöntemlerinde farklı eşik değerlerinin performans değerlendirilmesi amaçlanmıştır. Eşik değeri hesaplanırken, Wishart matrisleri için farklı olasılık dağılım fonksiyonları kullanılarak yanlış algılama olasılığı $\left(P_{f a}\right)$ ve eşik değeri teorik olarak verilmiştir. Benzetim çalışmaları, MIMO-OFDM sistemleri için gürültü belirsizliği altında gerçekleştirilmiştir. Ayrıca benzetim çalışması sonuçlarında en yaygın spektrum algılama yöntemlerinden olan enerji algılamaya da yer verilmektedir. Yapılan benzetim çalışmaları farklı gürültü seviyeleri için verilmektedir. Alınan sonuçlara göre iyileştirilmiş GLRD yönteminin başarılı sonuçlar verdiği gözlenmiştir.

Anahtar Kelimeler: Bilişsel Radyo, GLRD Eşik Değeri,„MIMO-OFDM Sistemleri, Tracy-Widom Dağılımı, Spektrum verimliliği,

\section{Multiple Antenna Spectrum Sensing Based on GLR Detector in Cognitive Radios}

\begin{abstract}
As it is known, eigenvalue-based spectrum detection methods are very preferred method for multi-antenna Cognitive Radio systems since they do not require any prior knowledge of the signal to be detected. In addition, eigenvalue based detection methods are generally the least affected by the noise uncertainty factor. In eigenvalue-based detection methods, detection performance depends on the correct calculation of the test statistics and the threshold value. In this study, it is aimed to evaluate the performance of different threshold values in generalized Likelihood Ratio Detection (GLRD) methods. While calculating the threshold value, misperception probability (Pfa) and threshold value are given theoretically using different probability distribution functions for Wishart matrices. Simulation studies were carried out under noise uncertainty for MIMO-OFDM systems. In addition, energy detection, which is one of the most common spectrum sensing methods, is included in the results of the simulation study. Simulation studies are given for different noise levels. According to the results, it is observed that the improved GLRD method gives successful results.
\end{abstract}

Keywords: Cognitive Radio, GLRD Threshold Value ,MIMO-OFDM Systems, Tracy-Widom Distribution, Spectrum efficiency

\footnotetext{
* Sorumlu Yazar: Erzincan Binali YILDIRIM Üniversitesi, Mühendislik Fakültesi, Elektrik Elektronik Müh. Bölümü, Erzincan, Türkiye, fyilgin@erzincan.edu.tr (ORCID: 0000-0002-7449-4811)
}

** Bu makale International Conference on Access to Recent Advances in Engineering and Digitalization (ARACONF 2020) de sunulmuştur. 


\section{Giriş}

Yapılan son ölçümler, frekans spektrumunun büyük bir bölümünün zamanın önemli bir bölümünde kullanılmadığını ortaya koymaktadır. Bu durumun en önemli nedeninin sabit spektrum atama yöntemleri olduğu bilinmektedir . Kablosuz iletişimde sunulan hizmet standardı ve bu hizmetlerden yararlanan kullanıcı sayısı giderek arttığından, sabit spektrum politikası artık sürdürülebilir olmaktan çıkmıştır. Günümüzde en önemli çözüm yöntemlerinden biri, Bilişsel Radyo (BR) sistemleridir. BR sistemlerinin amacı, frekans boşluklarını tespit etmek ve bu frekans boşluklarını lisansız ikincil kullanıcılara tahsis etmektir(Kortun, Ratnarajah, Sellathurai, Liang, \& Zeng, 2014) . Bu teknolojiyi uygulamanın en büyük zorluklarından biri, spektrumda birincil kullanıcı olup olmadığını en doğru şekilde belirlemektir. Bu zorlukla baş edebilmek için literatürde farklı yöntemler önerilmiştir. Filtre eşleş̧irme(QI, PENG, WANG, \& LUO, 2009), enerji algılama(Verma \& Singh, 2016), döngüsel durağanlık algılama(S \& Jayasheela, 2012), özdeğer tabanlı algılama(Çiflikli \& Ilgin, 2018) ve kovaryans tabanlı algılama(Wei, Tirkkonen, \& McKay, 1993), spektrum algılama için en yaygın kullanılan yöntemlerdendir. Bu yöntemler kendi içinde avantajlara ve dezavantajlara sahiptir. Örneğin, filtre denkleştirme yöntemi için algılanacak olan sinyal istatistiksel özellikleri bakımından tam olarak bilinmelidir (işaret varyansı, modülasyon türü vb.). Çevrimsel durağanlık algılama yöntemi sadece Dikgen Frekans Bölüşümlü Çoğullama (Orthogonal Frrquency Division Multiplexng-OFDM) tabanlı işaretlere uygulanabilir olması bu yöntemin dezavantajlarından biridir(Bandari, Vakamulla, \& Drosopoulos, 2018). Enerji algılama yöntemi hesaplama maliyeti açısından oldukça basit olsa da uygulamadaki zorlukları da beraberinde getirmektedir(Lavanya \& Bhagyaveni, 2019). Bu zorluklardan en önemlisi enerji algılama yönteminin başarılı bir algılama yapabilmesi için, ortamda varolan gürültü varyansının tam olarak bilinmesi gerekliliğidir. Enerji algılama tabanlı spektrum algılama performansı, özellikle gürültü belirsizliği altında, önemli ölçüde bozulabilir. Bazı algılama yöntemlerinde gürültü sinyali ve birincil kullanıcı sinyali hakkında önceden herhangi bir bilgi (varyans, modülasyon, kanal bilgisi) gerektirmediğinden uygulama açısından kolay olmaktadır. Bu yöntemlerin başında özdeğer tabanlı algılama yöntemleri gelmektedir. Ayrıca bu yöntemlerin çoğunda, test istatistiği ve eşik değeri, gürültü varyansından bağımsız olduğundan, bu yöntemler gürültü belirsizliği faktöründen önemli ölçüde etkilenmez. Literatürde özdeğer tabanlı algılama için farklı yöntemler önerilmiş̧ir. Maksimum Minimum Özdeğer (MMÖ)(Zeng \& Liang, 2009) ve Genelleştirilmiş En çok Olabilirlik Oranı (GEOO)(Liu, Z., Wang, 2017) gibi yöntemler performans bakımından önemi bir yer tutmaktadır. Bu yöntemler hesaplama maliyeti ve performansı açısından oldukça faydalı olsa da kovaryans matrisinin en büyük özdeğer dağılımı için uygun bir fonksiyon kullanılmadığından eşik değerleri doğru bir şekilde hesaplanamamıştır. Özellikle geleneksel yöntemle hesaplanan gürültü belirsizliği altındaki eşik değeri, bu yöntemlerde performansın düşmesine neden olmaktadır. Bu nedenle, özdeğer tabanlı algılama yöntemlerinde performans artışı sağlamak için eşik değerlerinin iyileştirilmesi gerekir.

$\mathrm{Bu}$ çalışmada toplanır beyaz Gauss gürültüsü ve Çok Giriş Çok Çıkışlı Sistemler (Multiple Input Multiple Output-MIMO) MIMO-OFDM sistemleri için Rayleigh sönümlenmeli kanal altında spektrum algılama sorunu incelenmektedir. Eşik değerinin belirlenmesi, spektrum algılamada algılayıcı performansını etkileyen en önemli faktörlerden biri olarak sayılabilir. Bu nedenle yapılacak çalışmalarda eşik değerinin doğru belirlenmesi çok önemlidir. Bu nedenle bu çalışmada GLRD tabanlı spektrum algılama yöntemleri için farklı eşik değerlerinin algılama performansları karşılaştırılmaktadır. Ayrıca benzetim sonuçlarında enerji tabanlı algılama için de ölçüm yapılmaktadır.

Bu çalışmanın organizasyonu şöyledir. İkinci bölümde, GLRD tabanlı spektrum algılama ve temel varsayımlar sunulmuştur. Üçüncü bölümde literatürde bulunan yeni eşik değeri ile, yanlış alarm olasılığı $\left(P_{f a}\right)$ ve algılama olasılığı $\left(P_{d}\right)$ teorik olarak hesaplanarak yarı kör bir GLRD tabanlı algılayıcı için teorik analizler yapılmaktadır. Dördüncü bölümde kör GLRD algılayıcısı için eşik değeri, $P_{f a}$ ve $P_{d}$ değerlerine teorik olarak yer verilmektedir. Benzetim çalışmaları beşinci bölümde, sonuçlar ise altıncı bölümde verilmiş̧ir.

\section{Materyal ve Metot}

\subsection{Genel Varsayımlar ve Spektrum Algılama}

Çok antenli BR sistemleri için Rayleigh sönümlü kanal altında $m$ adet ile donatılmış ikincil kullanıcıların spektrum algılama problemi göz önünde bulundurulmaktadır (Şekil 1). Buradaki başlıca hedef, birincil/lisanlı verici pasif olduğunda bu frekans bandını ikincil kullanıcılar için kullanılabilir hale getirmektir. Bu nedenle ikincil kullanıcıların bu spektrumu sürekli olarak tespit etmesi ve bu spektrumu lisanslı kullanıcı etkin olmadığında kullanması amaçlanmıştır. Birincil kullanıcı sinyali sıfır ortalamalı ve Gauss dağılıma sahip olduğunu varsayıyoruz. Böylece $\mathrm{H}_{0}$ da ilgili spektrumun boş olduğunu veya ikincil kullanıcıların spektrumun bu bölgesini firsatçı olarak kullanabileceğini belirtmektedir. $H_{l}$ ise bu spektrum bölgesinin dolu olduğunu göstermektedir. Bu temel iki varsayım matematiksel olarak Eşitlik (1) ve Eşitlik (2) 'de ifade edilmektedir(Kortun, Sellathurai, Ratnarajah, \& Zhong, 2012).

$$
\begin{array}{ll}
H_{0}: \boldsymbol{x}(\boldsymbol{n})=\boldsymbol{\eta}(\boldsymbol{n}) \\
H_{1}: \boldsymbol{x}(\boldsymbol{n})=\boldsymbol{s}(\boldsymbol{n})+\boldsymbol{\eta}(\boldsymbol{n})
\end{array}
$$

Burada $\boldsymbol{\eta}(\boldsymbol{n})$, sıfır ortalamalı Gauss dağılımlı gürültü işaretini, $\boldsymbol{s}(\boldsymbol{n})$, birincil kullanıcı sinyalini temsil etmektedir. $\boldsymbol{x}(\boldsymbol{n})$ ise m adet ikincil kullanıcı tarafından alınan işaretlerin bir matriste depolanmış biçimidir. Bu nedenle, sistemdeki ikincil kullanıcı sayısı $m$ olduğunda ve $n$ adet örnek alındığında, $\boldsymbol{x}(\boldsymbol{n})$ matrisi pxn boyutunda olmaktadır. Bu durumda, (3) ve (4) nolu eşitlikleri olasılık dağılım fonksiyonları açısından aşağıdaki gibi tanımlanmaktadır. 


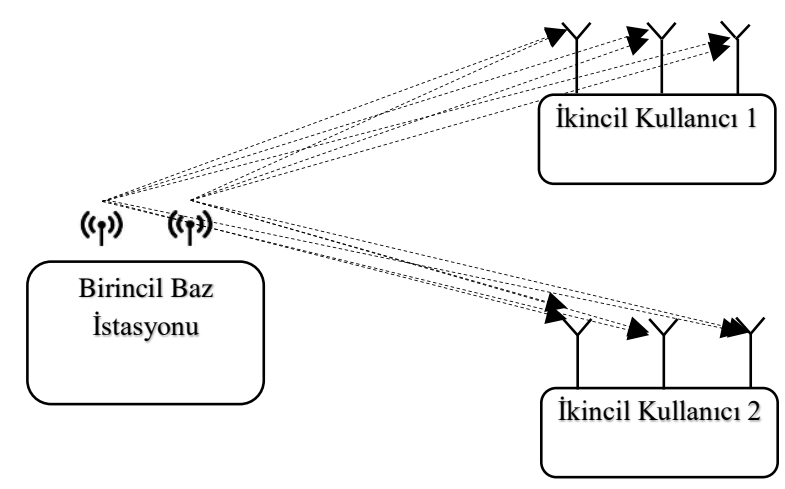

Şekil 1. Bilişsel Radyo Sistemleri için Önerilen Algılama Modeli

$H_{0}: \boldsymbol{x}(\boldsymbol{n}) \sim C N\left(0, \sigma_{n}^{2} I_{m}\right)$
$H_{1}: \boldsymbol{x}(\boldsymbol{n}) \sim \mathrm{CN}\left(0, \sigma_{x}^{2} h h^{H}+\sigma_{n}^{2} I_{m}\right)$

Burada $h \in \mathrm{C}^{m x 1}$, birincil kullanıcı ile $m$. anten arasındaki kanal katsayısı vektörünü, $\sigma_{x}^{2}$ ve $\sigma_{n}^{2}$ sırasıyla, birincil kullanıcı sinyali ve gürültüsünün varyansını göstermektedir. Böylece, her iki hipotez altında alınan sinyalin kovaryans matrisleri aşağıdaki gibi tanımlanmaktadır(Maaref \& Aïssa, 2007).

$$
\begin{array}{ll}
H_{0} & : \boldsymbol{R}_{\boldsymbol{\eta}}=\boldsymbol{\eta}(n) \boldsymbol{\eta}(n)^{\prime} \\
H_{1} & : \boldsymbol{R}_{\boldsymbol{x}}=\boldsymbol{x}(n) \boldsymbol{x}(n)^{\prime}
\end{array}
$$

Burada $\boldsymbol{R}_{\boldsymbol{\eta}}$, Wishart matrisidir. Bu matrislerin en büyük özdeğer dağılımı, son birkaç yılda, sayısal haberleşme de dahil olmak üzere birçok çalışmanın yapıldığı bir konudur. Wishart matrislerinin en büyük özdeğer dağılımı için ilk kapalı fonksiyon Jhonstone tarafından bulunmuş olsa da en doğru kapalı fonksiyon Deo tarafından elde edilmiştir(Deo, 2016a).

$\frac{\lambda_{\max } A(n)-\mu_{c}}{\sigma_{c}} \rightarrow T W_{1}$

Burada $\rightarrow T W_{1}$ ifadesi $m, p \rightarrow \infty$ koşulu ile bu eşitliğin 1. Dereceden Tracy-Widom dağılımına yakınsayacağını göstermektedir. Ayrıca $\lambda_{\max }$ kovaryans matrisi en büyük özdegerinin tanımlamaktadır. Bu eşitlikte kullanılan bazı parametreler Teorem 1 ile verilmektedir(Deo, 2016b).

Teorem 1: Gürültünün karmaşık olduğunu varsayılsın.

$\boldsymbol{A}(n)=\frac{n}{\sigma_{\eta}^{2}} \boldsymbol{R}_{\boldsymbol{\eta}}, \quad \mu_{n, m}=n^{-1}\left(\sqrt{n-\frac{1}{2}}+\sqrt{m-1 / 2}\right)^{2}, \sigma_{n, m}=\left(\mu_{n, m} / n\right)^{1 / 2}\left(\frac{1}{\sqrt{n-1 / 2}}+\frac{1}{\sqrt{m-1 / 2}}\right)^{1 / 3}, \mu_{c}=\mu_{n, m}+a \sigma_{n, m} \quad$ ve $\sigma_{c}=$ $\sqrt{\frac{n m}{2+n m}\left(\sigma_{n, m, 0}^{2}-\mu_{c}^{2}\right)}$ olsun. Burada $a, b$ ve sırasıyla $-1.206548,1.267941$ 'e eşittir. Ve $\sigma_{n, m, 0}=b \sigma_{n, m}$ anlamına gelmektedir. Bu nedenle $\frac{\lambda_{\max } A(n)-\mu_{c}}{\sigma_{c}}$, birinci dereceden Tracy-Widom dağılımına yakınsamaktadır.

Spektrum algılama uygulamalarında genellikle bir test istatistiği eşik değeri ile karşılaştırılıp buna göre spektrum kararı verilmektedir. Yarı-kör GLRD dedektörü için Neyman-Pearson kuralına göre test istatistiği ve algılama kuralı aşağıda belirtilmiştir(He, Ratnarajah, Yousif, Xue, \& Sellathurai, 2016).

$\frac{\lambda_{\max }}{\sigma_{n}^{2}} \gtrless_{H_{0}}^{H_{1}} \gamma_{y k}$

Burada $\frac{\lambda_{\max }}{\sigma_{n}^{2}}$ ve $\gamma$ sırası ile Test İstatistiğini (TI) ve eşik değerinin gösterir. Dikkat edilirse, test istatistiği gürültü varyansına bağlıdır. Bu nedenle, bu yöntem yarı kör yöntem olarak tanımlanmaktadır. Yöntemin doğru kararı verme yeteneği, gürültü gücü tahminine ve 
eşik değerine bağlıdır. Kör GLRD yöntemine gelince, test istatistiği yalnızca alınan sinyalin özdeğerlerine bağlıdır (gürültü gücünün tahmini gerekli değildir). Bu nedenle, yöntem kör algılama olarak bilinmektedir. Kör GLRD için test istatistiği aşağıdaki gibi tanımlanmaktadır.

$\frac{\lambda_{\max }}{\sum_{i=2}^{m} \lambda_{i}} \gtrless_{H_{0}}^{H_{1}} \gamma_{k}$

\subsection{Yarı-Kör GLRD dedektörü İçin Eşik Değerinin Hesaplanması}

Bu bölümde yarı kör GLRD için eşik değeri elde edilmektedir. Eşitlik (7) ve Eşitlik (8) 'den görülebileceği gibi eşiğin doğruluğu yöntemin başarısını doğrudan etkiler. Algılama teorisine göre yanlış alarm olasıllığı ve algılama olasıllığı, aşağıdaki koşullu olasilıklarla tanımlanmaktadır(Abbas Taherpour, Masoumeh Nasiri-Kenari, 2010).

$$
\begin{gathered}
P_{f a}=P\left(T S>\gamma_{y k} \mid H_{0}\right) \\
P_{d}=P\left(T S>\gamma_{y k} \mid H_{1}\right)
\end{gathered}
$$

Burada $\gamma_{y k}$, yarı-kör GLRD dedektörü için eşik değerini belirtir. Böylece yarı kör GLRD için Pfa aşağıdaki şekilde elde edilmektedir.

$$
P_{f a}=P\left(\frac{\lambda_{\max }\left(R_{\eta}\right)}{\sigma_{n}^{2}}>\gamma_{y k}\right)
$$

Eşik değerini elde etmek için eşitliğin her iki tarafına $n$ eklendiğinde, bir taraf birinci dereceden Tracy-Widom dağılımına benzemektedir.

$P_{f a}=P\left(\lambda_{\max }\left(R_{\eta}\right) \frac{n}{\sigma_{\eta}^{2}}>\gamma_{y k} n\right)$

Ardından Eşitlik (10) 'da $\frac{n}{\sigma_{\eta}^{2}} R_{\eta}$ yerine A(n) yazıldığında Eşitlik (14) elde edilmektedir.

$\left.P_{f a}=P\left(\lambda_{\max }(A(n))>\gamma_{y k} n\right)\right)$

Eşitlik 14'ü birinci dereceden Tracy-Widom dağılımına benzetmek için gerekli eklemeler eşitsizliğin her iki tarafına yapılmalıdır.

$P_{f a}=P\left(\left(\frac{\lambda_{\max } A(n)-\mu_{c}}{\sigma_{c}}\right)>\left(\frac{\gamma_{y k} n-\mu_{c}}{\sigma_{c}}\right)\right)$

Survival fonksiyonu kullanılarak Eşitlik (15) Eşitlik (16)'ya dönüşmektedir .

$$
P_{f a}=1-F_{1}\left(\frac{\gamma_{y k} n-\mu_{c}}{\sigma_{c}}\right)
$$

$F_{1}^{-1}\left(1-P_{f a}\right)=\left(\frac{\gamma_{y k} n-\mu_{c}}{\sigma_{c}}\right)$

$F_{1}^{-1}\left(1-P_{f a}\right)=\left(\frac{\gamma_{y k} n-\left(\mu_{n, m}+a \sigma_{n, m}\right)}{\sqrt{\frac{n p}{2+n p}\left(\sigma_{n, m, 0}^{2}-\mu_{c}^{2}\right)}}\right)$

Son olarak, eşik değeri aşağıdaki gibi elde edilir(Çiflikli \& Ilgin, 2018).

$\gamma_{y k}=\frac{F_{1}^{-1}\left(1-P_{f a}\right) \sqrt{\frac{n p}{2+n p}\left(\sigma_{n, m, 0}^{2}-\mu_{c}^{2}\right)}}{n}+\frac{\left(\mu_{n, m}+a \sigma_{n, m}\right)}{n}$ 
Burada $F_{1}^{-1}$, 1.Dereceden Tracy-Widom dağılımını belirtir. Bu dağılım için bazı değerler Tablo 1'de verilmiştir.

Tablo 1. Birinci Dereceden Tracy-Widom Dă̆ılımı için Sayısal Değerler.

\begin{tabular}{|c|c|c|c|c|c|}
\hline$x$ & -3.90 & -2.78 & -1.27 & 0.45 & 2.02 \\
\hline$F_{l}(x)$ & 0.01 & 0.10 & 0.50 & 0.90 & 0.99 \\
\hline
\end{tabular}

\subsection{Kör GLRD Dedektörü İçin Eşik Değerinin Hesaplanması}

Kör GLRD için $P_{f a}$ aşağıdaki gibi hesaplanmaktadır.

$P_{f a}=P\left(\frac{\lambda_{\max }\left(\boldsymbol{R}_{\boldsymbol{\eta}}\right)}{\sum_{i=2}^{m} \lambda_{i}}>\gamma_{k}\right)$

Burada $\gamma_{k}$ kör GLRD dedektörü için eşik değerini belirtir. $\sum_{i=2}^{m} \lambda_{i} \approx(m-1) \sigma_{\eta}^{2}$ olduğundan, denklem aşağıdaki gibi düzenlenebilir.

$$
\begin{aligned}
& \left.P_{f a}=P\left(\lambda_{\max }\left(\boldsymbol{R}_{\boldsymbol{\eta}}\right)>\gamma_{k} \sum_{i=2}^{m} \lambda_{i}\right)\right) \\
& \left.P_{f a}=P\left(\frac{\lambda_{\max }\left(\boldsymbol{R}_{\boldsymbol{\eta}}\right)}{\sigma_{\eta}^{2}}>\gamma_{k}(m-1)\right)\right)
\end{aligned}
$$

Denklemin bir tarafı birinci dereceden Tracy-Widom dağılımına dönştürülmelidir.

$P_{f a}=P\left(\lambda_{\max }\left(\boldsymbol{R}_{\boldsymbol{\eta}}\right) \frac{n}{\sigma_{\eta}^{2}}>\gamma_{k} n(m-1)\right)$

$P_{f a}=P\left(\left(\frac{\lambda_{\max } A(n)-\mu_{c}}{\sigma_{c}}\right)>\left(\frac{\left(\gamma_{k} n(m-1)\right)-\mu_{c}}{\sigma_{c}}\right)\right)$

$P_{f a}=1-\mathrm{F}\left(\frac{\left(\gamma_{k}(m-1) n\right)-\mu_{c}}{\sigma_{c}}\right)$

$F_{1}^{-1}\left(1-P_{f a}\right)=\frac{\left(\gamma_{k}(m-1) n\right)-\mu_{c}}{\sigma_{c}}$

$F_{1}^{-1}\left(1-P_{f a}\right) \sigma_{c}+\mu_{c}=\gamma_{k}(m-1) n$

En son olarak, eşik değeri aşağıdaki şekilde elde edilir(Çiflikli \& Ilgin, 2018).

$\gamma_{k}=\frac{F_{1}^{-1}\left(1-P_{f a}\right) \sigma_{c}+\mu_{c}}{(m-1) n}$

Eşitlik (19) ve eşitlik (28) karşılaştırıldığında yarı kör GLRD tabanlı yöntemde eşik değerinde $\sigma_{\mathrm{n}, \mathrm{m}, 0}^{2}$ ifadesi olduğu görülmektedir. Bu ifade eşik değeri hesaplanması için gürültü varyansının bilinmesi gerektiğini göstermektedir. Fakat eşitlik (28)'de eşik değerinin hesaplanması için gürültü varyansına ihtiyaç olmadığı görülmektedir.

\section{Araştırma Sonuçları ve Tartışma}

\subsection{Bulgular}

GLRD tabanlı algılama yöntemlerinin performansınlarını görmek için bu bölümde benzetim sonuçları verilmiştir. Simülasyonlarda MIMO-OFDM tabanlı haberleşme sistemi kullanılmıştır. GLRD tabanlı algılama yöntemlerinin 2x4 MIMO sistemi için değişen SNR değerlerine karşı algılama performansları Şekil 2.' de verilmektedir. $P_{f a} 0.1$ olarak seçilmiştir (bu WRAN 802.22 çalışma grubunun izin verdiği sınır değerdir). Sonuçların daha doğru yorumlanması için farklı eşik değeri kullanan GLRD tabanlı algılama yöntemleri karşılaştırılmıştır. 


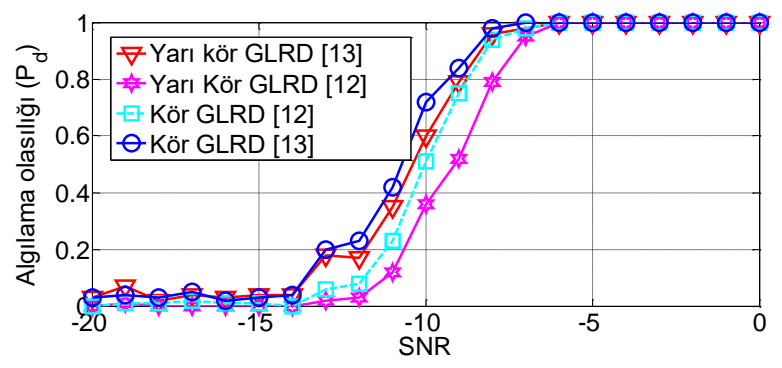

Şekil 2. 2x4 MIMO-OFDM Sistemi için $P_{d^{-}}$SNR Grafiği, $n=500$

Şekil 2'den görüldüğü üzere bu çalışmada açıklanan eşik değeri kullanıldığında ve geleneksel eşik değeri kullanıldığında GLRD tabanlı algılama yöntemlerinin algılama performanslarında önemli ölçüde fark gözlemlenmektedir.

Şekil 3'te ise Şekil 2'den farklı olarak örnek sayısı 1000 seçilmiştir. BR sistemlerinde algılama süresi oldukça önemlidir. Belirlenen spektrum bölgesi en kısa sürede algılanmalı ve lisanslı kullanıcı tekrar hatta girdiğinde bu bölge boşaltılmalıdır. Bu nedenle algılama işlemi en kısa sürede en az örnek sayısı ile yapılmalıdır. Aksi durumlar lisanlı kullanıcıların spektruma erişimini kısıtlayacă̆ından büyük hukuki sorumluluklar doğurabilmektedir. Ayrıca Şekil 3’te enerji tabanlı algılama sonucuna da yer verilmektedir. Görüldüğü üzere enerji tabanlı algılama GLRD yönteminden daha başarısız bir algılama gerçekleştirmektedir.

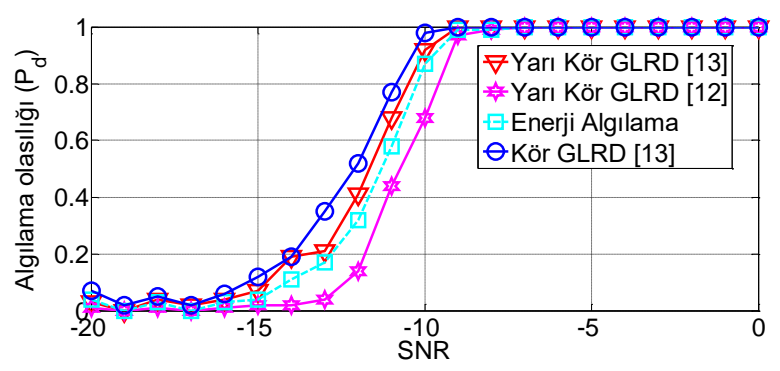

Şekil 3. 2x4 MIMO-OFDM Sistemi için $P_{d^{-}}$SNR Grafiği, $n=1000$

\section{Sonuç}

Bu çalışmada GLRD tabanlı spektrum algılama yöntemleri için farklı eşik değerleri kullanıldığında spektrum algılama performansları ölçülmektedir. Eşik değeri hesaplanırken, yanlış algılama olasılığı $\left(P_{f a}\right)$ ve algılama olasılığı Wishart matrisleri için yeni bir olasılık dağılım fonksiyonu kullanılarak geri kazanılmıştır. Simülasyonlar, MIMO-OFDM sistemleri için gürültü belirsizliği altında yapılmıştır ve geleneksel yöntemlerle karşılaştırıldığında yeni eşik değeri ile belirgin bir performans artışı gözlemlenmiştir.

\section{Kaynakça}

Abbas T., Masoumeh Nasiri-Kenari, S. G. (2010). Multiple antenna spectrum sensing in cognitive radios. IEEE TRANSACTIONS ON WIRELESS COMMUNICATIONS, 9(2), 814-823.

Bandari, S. K., Vakamulla, V. M., \& Drosopoulos, A. (2018). GFDM/OQAM performance analysis under Nakagami fading channels. Physical Communication, 26, 162-169. https://doi.org/10.1016/J.PHYCOM.2017.12.008

Çiflikli, C., \& Ilgin, F. Y. (2018). Covariance Based Spectrum Sensing with Studentized Extreme Eigenvalue. Technical Gazette, 25(6), 100-106.

Deo, R. S. (2016a). On the Tracy-Widom approximation of studentized extreme eigenvalues of Wishart matrices. Journal of Multivariate Analysis, 147, 265-272. https://doi.org/10.1016/j.jmva.2016.01.010

Deo, R. S. (2016b). On the Tracy-Widom approximation of studentized extreme eigenvalues of Wishart matrices. Journal of Multivariate Analysis, 147, 265-272. https://doi.org/10.1016/j.jmva.2016.01.010

He, Y., Ratnarajah, T., Yousif, E. H. G., Xue, J., \& Sellathurai, M. (2016). Performance analysis of multi-antenna GLRT-based spectrum sensing for cognitive radio. Signal Processing, 120, 580-593. https://doi.org/10.1016/j.sigpro.2015.10.018

Kortun, A., Ratnarajah, T., Sellathurai, M., Liang, Y. C., \& Zeng, Y. (2014). On the eigenvalue-based spectrum sensing and secondary user throughput. IEEE Transactions on Vehicular Technology, 63(3), 1480-1486. https://doi.org/10.1109/TVT.2013.2282344

Kortun, A., Sellathurai, M., Ratnarajah, T., \& Zhong, C. (2012). Distribution of the ratio of the largest eigenvalue to the trace of complex wishart matrices. IEEE Transactions on Signal Processing, 60(10), 5527-5532. 
https://doi.org/10.1109/TSP.2012.2205922

Lavanya, S., \& Bhagyaveni, M. A. (2019). EVM based rate maximized relay selection for cooperative cognitive radio networks. AEU - International Journal of Electronics and Communications, 104, 86-90. https://doi.org/10.1016/j.aeue.2018.12.018

Liu, Z., Wang, Y. (2017). A note on spiked Wishart matrices. Statistics and Probability Letters, 127, 1-6. https://doi.org/10.1017/CBO9781107415324.004

Maaref, A., \& Aïssa, S. (2007). Eigenvalue distributions of wishart-type random matrices with application to the performance analysis of MIMO MRC systems. IEEE Transactions on Wireless Communications, 6(7), 2678-2689. https://doi.org/10.1109/TWC.2007.05990

QI, Y., PENG, T., WANG, W., \& LUO, S. (2009). Cyclostationary signature design for common control channel of cognitive radio. The Journal of China Universities of Posts and Telecommunications, 16(2), 42-46. https://doi.org/10.1016/S10058885(08)60202-2

S, A. P., \& Jayasheela, M. (2012). Cyclostationary feature detection in cognitive radio using different modulation schemes. International Journal of Computer Applications, 47(21), 975-8887. https://doi.org/10.7763/IJFCC.2013.V2.249

Verma, P., \& Singh, B. (2016). Overcoming sensing failure problem in double threshold based cooperative spectrum sensing. Optik, 127(10), 4200-4204. https://doi.org/10.1016/j.ijleo.2016.01.108

Wei, L., Tirkkonen, O., \& McKay, M. R. (1993). Exact demmel condition number distribution of complex wishart matrices via the mellin transform. IEEE Transactions on Aerospace and Electronic Systems, 29(3), 834-840. https://doi.org/10.1109/7.220934

Zeng, Y., \& Liang, Y. C. (2009). Spectrum-sensing algorithms for cognitive radio based on statistical covariances. IEEE Transactions on Vehicular Technology, 58(4), 1804-1815. https://doi.org/10.1109/TVT.2008.2005267 